\title{
ON THE RATE OF THE VOLUME GROWTH FOR SYMMETRIC VISCOUS HEAT-CONDUCTING GAS FLOWS WITH A FREE BOUNDARY
}

\author{
ALEXANDER ZLOTNIK
}

Received 6 June 2005; Accepted 10 July 2005

The system of quasilinear equations for symmetric flows of a viscous heat-conducting gas with a free external boundary is considered. For global in time weak solutions having nonstrictly positive density, the linear in time two-sided bounds for the gas volume growth are established.

Copyright (c) 2006 Alexander Zlotnik. This is an open access article distributed under the Creative Commons Attribution License, which permits unrestricted use, distribution, and reproduction in any medium, provided the original work is properly cited.

\section{Introduction}

We consider the system of quasilinear equations describing symmetric flows of a viscous heat-conducting perfect polytropic gas [1]

$$
\begin{gathered}
\eta_{t}=\left(r^{k} v\right)_{x}, \\
v_{t}=r^{k} \sigma_{x}, \\
c_{V} \theta_{t}=\left(r^{k} \pi\right)_{x}+\sigma\left(r^{k} v\right)_{x}-2 k \mu\left(r^{k-1} v^{2}\right)_{x}, \\
r_{t}=v, \\
\sigma=v \rho\left(r^{k} v\right)_{x}-R \rho \theta, \quad \pi=\varkappa \rho r^{k} \theta_{x}, \quad \rho=\frac{1}{\eta},
\end{gathered}
$$

in the domain $Q:=\Omega \times \mathbb{R}^{+}=(0, M) \times(0, \infty)$. The system is supplemented with the boundary and initial conditions

$$
\begin{gathered}
\left.v\right|_{x=0}=0,\left.\quad\left(\sigma-2 k \mu \frac{v}{r}\right)\right|_{x=M}=0,\left.\quad\left(r^{k} \pi\right)\right|_{x=0, M}=0 \quad \text { for } t>0, \\
\left.\{\eta, v, \theta, r\}\right|_{t=0}=\left\{\eta^{0}(x), v^{0}(x), \theta^{0}(x), r^{0}(x)\right\} \quad \text { for } x \in \Omega .
\end{gathered}
$$

The parameter $k$ takes the values 1 or 2 accordingly to the cylindrical or spherical symmetry. 
The unknown functions $\eta>0, v, \theta \geq 0$ and $r \geq r_{0}$ depend on the Lagrangian mass coordinates $(x, t)$ and denote the specific volume, the velocity, the absolute temperature and the Eulerian coordinate that is the radius of a gas particle. The functions $\rho, \sigma$ and $-\pi$ are the density, the stress and the heat flux. We consider flows around a hard core so that $r_{0}>0$ is its radius, and the internal boundary $(x=0)$ is one with the core. The external boundary $(x=M)$ is free; both boundaries are thermally isolated, see (1.6).

The quantities $\nu>0, \mu, R>0, c_{V}>0$ and $\varkappa>0$ are physical constants; $M>0$ is the total mass of the gas. We impose the standard condition on the viscosity coefficients $\nu$ and $\mu$

$$
v_{1}:=v-\frac{2 k}{k+1} \mu>0
$$

The initial function $r^{0}$ is not arbitrary but rather connected to $\eta^{0}$ by the physical relation

$$
\left(r^{0}\right)^{k+1}(x)=r_{0}^{k+1}+(k+1) \int_{0}^{x} \eta^{0}(\xi) d \xi \quad \text { for } x \in \bar{\Omega}
$$

In the simpler case of the planar symmetry $(k=0)$, the asymptotic behavior of solutions was studied in detail in [5] and more recently in $[6,7]$ for other boundary conditions. In the case of the spherical symmetry, some results on the growth of the (scaled) gas volume $V(t):=\int_{\Omega} \eta(x, t) d x$ as $t \rightarrow \infty$ are available in [2].

We prove the sharp result establishing the linear growth of $V$ both in the cases of the spherical and cylindrical symmetries like that for the planar one. In contrast to [2, 57], we treat essentially more general global in time weak solutions to the problem whose density is non-strictly positive only.

\section{Results}

We introduce the integration operators

$$
I z(x):=\int_{0}^{x} z(\xi) d \xi, \quad I^{*} z(x):=\int_{x}^{M} z(\xi) d \xi \quad \text { for } z \in L^{1}(\Omega) .
$$

They are connected by the identity

$$
\int_{\Omega}\left(I z_{1}\right) z_{2} d x=\int_{\Omega} z_{1} I^{*} z_{2} d x \text { for any } z_{1}, z_{2} \in L^{1}(\Omega) .
$$

Let $V_{q}\left(Q_{T}\right)$ be the space of functions $w \in L^{q, \infty}\left(Q_{T}\right)$ having the derivative $w_{x} \in L^{q}\left(Q_{T}\right)$, for $q=1,2$ and $Q_{T}:=\Omega \times(0, T)$; recall that $\|w\|_{L^{q, s}\left(Q_{T}\right)}=\|\| w\left\|_{L^{q}(\Omega)}\right\|_{L^{s}(0, T)}$, for $q, s \in[1, \infty]$.

We study global in time weak solution to the problem (1.1)-(1.7) such that:

(1) the properties

$$
\begin{gathered}
\eta, \eta_{t} \in L^{1,2}\left(Q_{T}\right), \quad \frac{1}{\eta} \in L^{\infty}\left(Q_{T}\right), \quad v \in V_{2}\left(Q_{T}\right), \\
\theta \in V_{1}\left(Q_{T}\right), \quad r, r_{x}, r_{t} \in L^{1, \infty}\left(Q_{T}\right)
\end{gathered}
$$

together with $\eta>0, \theta \geq 0, r \geq r_{0}$ (almost everywhere in $Q_{T}$ ) and $\left.v\right|_{x=0}=0$ are valid; 
(2) equations (1.1) and (1.4) together with the initial conditions $\left.\eta\right|_{t=0}=\eta^{0}$ and $\left.r\right|_{t=0}=$ $r^{0}$ are satisfied;

(3) the integral identities

$$
\int_{Q_{T}}\left\{-v \varphi_{t}+\sigma\left(r^{k} \varphi\right)_{x}\right\} d x d t=\left.\int_{\Omega} v^{0} \varphi\right|_{t=0} d x+\left.\left.2 k \mu \int_{0}^{T}\left(v r^{k-1}\right)\right|_{x=M} \varphi\right|_{x=M} d t
$$

for any $\varphi \in H^{1}\left(Q_{T}\right)$ with $\left.\varphi\right|_{x=0}=0$ and $\left.\varphi\right|_{t=T}=0$, as well as

$$
\int_{Q_{T}}\left\{-c_{V} \theta \psi_{t}+r^{k} \pi \psi_{x}-\left[\sigma\left(r^{k} v\right)_{x}-2 k \mu\left(r^{k-1} v^{2}\right)_{x}\right] \psi\right\} d x d t=\left.\int_{\Omega} c_{V} \theta^{0} \psi\right|_{t=0} d x,
$$

for any $\psi \in C^{1}\left(\bar{Q}_{T}\right)$ with $\left.\psi\right|_{t=T}=0$, are valid, where relations (1.5) are assumed to hold.

Hereafter $T>0$ is arbitrary and it is assumed that $\eta^{0} \in L^{1}(\Omega), v^{0} \in L^{2}(\Omega), \theta^{0} \in L^{1}(\Omega)$ as well as $\eta^{0}>0$ and $\theta^{0} \geq 0$ (almost everywhere in $\Omega$ ).

We have to justify correctness of the definition of the weak solution. First notice that actually $\eta \in L^{1, \infty}\left(Q_{T}\right)$ and $r \in L^{\infty}\left(Q_{T}\right)$ according to properties (2.3). Next, we recall that (1.1) and (1.4) together with relation (1.9) imply the following relation between $r$ and $\eta$

$$
r^{k+1}=r_{0}^{k+1}+(k+1) I \eta \text {. }
$$

In particular, actually $r \geq r_{0}$ and $\rho r_{x}=r^{-k}$. Consequently

$$
\sigma=\rho\left(v r^{k} v_{x}-R \theta\right)+v k r^{-1} v \in L^{2}\left(Q_{T}\right),
$$

where the embedding $V_{1}\left(Q_{T}\right) \subset L^{2}\left(Q_{T}\right)$ is taken into account. Moreover, for any $\varphi \in$ $V_{2}\left(Q_{T}\right)$, we have

$$
\sigma\left(r^{k} \varphi\right)_{x}=\sigma r^{k} \varphi_{x}+k r^{-1}\left(\nu r^{k} v_{x}-R \theta\right) \varphi+v k^{2} r^{k-2} r_{x} v \varphi,
$$

and since $V_{2}\left(Q_{T}\right) \subset L^{\infty, 4}\left(Q_{T}\right)[4]$, we obtain

$$
\sigma\left(r^{k} \varphi\right)_{x} \in L^{1}\left(Q_{T}\right) .
$$

If in addition $\varphi_{x} \in L^{2, \infty}\left(Q_{T}\right)$, then

$$
\sigma\left(r^{k} \varphi\right)_{x} \in L^{1,2}\left(Q_{T}\right)
$$

Furthermore

$$
\left(r^{k-1} v^{2}\right)_{x}=2 r^{k-1} v v_{x}+(k-1) r^{k-2} r_{x} v^{2} \in L^{1,2}\left(Q_{T}\right) .
$$

Consequently identities (2.4) and (2.5) are well-defined.

Notice also that

$$
\sigma \eta=v r^{k} v_{x}-R \theta+\nu k r^{-1} v \eta \in L^{1,2}\left(Q_{T}\right) .
$$

Concerning the existence of strong and weak solutions, see in particular $[1,3,8]$. 
4 The rate of the volume growth

We will need the energy conservation law. Let us set $\sigma_{\Gamma}:=\left.2 k \mu(v / r)\right|_{x=M}$; notice that $\sigma_{\Gamma} \in L^{4}(0, T)$.

Lemma 2.1. The total kinetic energy $(1 / 2) \int_{\Omega} v^{2} d x$ and the total internal energy $\int_{\Omega} c_{V} \theta d x$ are absolutely continuous functions on $[0, T]$ for any $T>0$ having the derivatives

$$
\frac{d}{d t} \frac{1}{2} \int_{\Omega} v^{2} d x=-\int_{\Omega}\left(\sigma-\sigma_{\Gamma}\right)\left(r^{k} v\right)_{x} d x, \quad \frac{d}{d t} \int_{\Omega} c_{V} \theta d x=\int_{\Omega}\left(\sigma-\sigma_{\Gamma}\right)\left(r^{k} v\right)_{x} d x
$$

Consequently the total energy conservation law holds

$$
\mathscr{E}:=\int_{\Omega}\left(\frac{1}{2} v^{2}+c_{V} \theta\right) d x \equiv \mathscr{E}^{0} \quad \text { on } \overline{\mathbb{R}}^{+}
$$

where $\mathscr{E}^{0}:=\int_{\Omega}\left((1 / 2)\left(v^{0}\right)^{2}+c_{V} \theta^{0}\right) d x$ is the total initial energy.

Proof. Though results of the stated type are known, we prefer to present an independent proof.

(1) We first notice that if a function $w \in L^{2}\left(Q_{T}\right)$ has the derivatives $w_{x},\left(I^{*} w\right)_{t} \in$ $L^{2}\left(Q_{T}\right)$ and $\left.w\right|_{x=0}=0$, then the function $\int_{\Omega} w^{2} d x$ is absolutely continuous on $[0, T]$ and has the derivative

$$
\frac{d}{d t} \int_{\Omega} w^{2} d x=2 \int_{\Omega}\left(I^{*} w\right)_{t} w_{x} d x
$$

Actually, under the additional condition $w_{t} \in L^{2}\left(Q_{T}\right)$, by exploiting identity (2.2) we have

$$
2 \int_{t_{1}}^{t_{2}} \int_{\Omega}\left(I^{*} w\right)_{t} w_{x} d x d t=\left.\int_{\Omega} w^{2} d x\right|_{t_{1}} ^{t_{2}}
$$

for all $0 \leq t_{1} \leq t_{2} \leq T$. In the general case, by applying (2.16) for $w$ mollified with respect to $t$ and passing to the limit there, we establish (2.16) for almost all $t_{1}$ and $t_{2}$ such that $0 \leq t_{1} \leq t_{2} \leq T$. This leads to (2.15).

(2) We rewrite identity (2.4) in the form

$$
\int_{Q_{T}}\left\{-v \varphi_{t}+\left(\sigma-\sigma_{\Gamma}\right)\left(r^{k} \varphi\right)_{x}\right\} d x d t=\left.\int_{\Omega} v^{0} \varphi\right|_{t=0} d x
$$

Since $\left(r^{k} \varphi\right)_{x}=r^{k} \varphi_{x}+\left(r^{k}\right)_{x} \varphi$, by choosing $\varphi:=I \zeta$ with $\zeta \in C^{1}\left(\bar{Q}_{T}\right)$ having $\left.\zeta\right|_{t=0, T}=0$ and applying (2.2), we get

$$
\int_{Q_{T}}\left\{-\left(I^{*} v\right) \zeta_{t}+\left(\sigma-\sigma_{\Gamma}\right) r^{k} \zeta+\left\{I^{*}\left[\left(\sigma-\sigma_{\Gamma}\right)\left(r^{k}\right)_{x}\right]\right\} \zeta\right\} d x d t=0
$$


Thus by definition there exists the weak derivative

$$
\left(I^{*} v\right)_{t}=-\left(\sigma-\sigma_{\Gamma}\right) r^{k}-I^{*}\left[\left(\sigma-\sigma_{\Gamma}\right)\left(r^{k}\right)_{x}\right] \in L^{2}\left(Q_{T}\right)
$$

see properties (2.7) and (2.10) for $\varphi \equiv 1$. By integrating over $\Omega$ this equality multiplied by $v_{x}$ we have

$$
\int_{\Omega}\left(I^{*} v_{t}\right) v_{x} d x=-\int_{\Omega}\left\{\left(\sigma-\sigma_{\Gamma}\right) r^{k} v_{x}+\left(\sigma-\sigma_{\Gamma}\right)\left(r^{k}\right)_{x} v\right\} d x=-\int_{\Omega}\left(\sigma-\sigma_{\Gamma}\right)\left(r^{k} v\right)_{x} d x,
$$

where property (2.9) for $\varphi=v$ is also taken into account. This together with formula (2.15) imply the first formula (2.13).

The second formula (2.13) arises simpler after choosing $\psi \in C^{1}[0, T]$ with $\left.\psi\right|_{t=0, T}=0$ in identity (2.5).

Let us establish the key equality in the paper. We set $V_{0}:=r_{0}^{k+1} /(k+1)$.

Lemma 2.2. The following equality holds

$$
\frac{d W}{d t}=\int_{\Omega}\left\{\frac{1}{k+1}\left[1+k\left(\frac{r_{0}}{r}\right)^{k+1}\right] v^{2}+R \theta\right\} d x
$$

where the function

$$
W:=\nu_{1} V+\frac{2 k}{k+1} \mu V_{0} \log \left(V_{0}+V\right)+\int_{\Omega} \frac{v}{r^{k}} \operatorname{I\eta } d x
$$

is absolutely continuous on $[0, T]$ for any $T>0$.

Proof. Equation (1.1) and the definition of $\sigma$ imply

$$
\nu \eta_{t}=\sigma \eta+R \theta=\sigma_{\Gamma} \eta+\left(\sigma-\sigma_{\Gamma}\right) \eta+R \theta .
$$

By integrating this equality over $\Omega$ we get

$$
\nu \frac{d V}{d t}=\sigma_{\Gamma} V+\int_{\Omega}\left(\sigma-\sigma_{\Gamma}\right) \eta d x+\int_{\Omega} R \theta d x .
$$

Let us transform the first and second summands in the right-hand side. By integrating (1.1) over $\Omega$ we get

$$
\frac{d V}{d t}=\left.\left(r^{k} v\right)\right|_{x=M}
$$

Using this equality together with (2.6) for $x=M$, we obtain

$$
\sigma_{\Gamma} V=2 k \mu \frac{\left.\left(r^{k} v\right)\right|_{x=M}}{\left.r^{k+1}\right|_{x=M}} V=\frac{2 k}{k+1} \mu \frac{V}{V_{0}+V} \frac{d V}{d t}=\frac{2 k}{k+1} \mu \frac{d}{d t}\left[V-V_{0} \log \left(V_{0}+V\right)\right] .
$$


6 The rate of the volume growth

Let $\zeta \in C^{1}\left(\bar{Q}_{T}\right)$ and $\left.\zeta\right|_{t=0, T}=0$. By choosing $\varphi:=I \zeta / r^{k}$ in identity (2.17), using the formula

$$
\left(\frac{I \zeta}{r^{k}}\right)_{t}=\frac{I \zeta_{t}}{r^{k}}-k \frac{I \zeta}{r^{k+1}} v
$$

(see (1.4)) and applying identity (2.2), we find

$$
\int_{Q_{T}}\left\{-\left(I^{*} \frac{v}{r^{k}}\right) \zeta_{t}+k\left(I^{*} \frac{v^{2}}{r^{k+1}}\right) \zeta+\left(\sigma-\sigma_{\Gamma}\right) \zeta\right\} d x d t=0
$$

This means that there exists the derivative

$$
\left(I^{*} \frac{v}{r^{k}}\right)_{t}=-k I^{*}\left(\frac{v^{2}}{r^{k+1}}\right)-\left(\sigma-\sigma_{\Gamma}\right) \in L^{2}\left(Q_{T}\right) .
$$

Moreover, $\left(I^{*}\left(v / r^{k}\right)\right)_{t} \eta \in L^{1,2}\left(Q_{T}\right)$ according to property (2.12). By integrating over $\Omega$ the last equality multiplied by $\eta$ we have

$$
\int_{\Omega}\left(\sigma-\sigma_{\Gamma}\right) \eta d x=-\frac{d}{d t} \int_{\Omega}\left(I^{*} \frac{v}{r^{k}}\right) \eta d x+\int_{\Omega}\left(I^{*} \frac{v}{r^{k}}\right) \eta_{t} d x-\int_{\Omega} k I^{*}\left(\frac{v^{2}}{r^{k+1}}\right) \eta d x
$$

Therefore by applying identity (2.2), equalities $I \eta_{t}=r^{k} v$ and $I \eta=\left(r^{k+1} /(k+1)\right)-$ $\left(r_{0}^{k+1} /(k+1)\right)$, see $(1.1)$ and $(2.6)$, we obtain

$$
\int_{\Omega}\left(\sigma-\sigma_{\Gamma}\right) \eta d x=-\frac{d}{d t} \int_{\Omega} \frac{v}{r^{k}} I \eta d x+\int_{\Omega}\left\{\frac{1}{k+1} v^{2}+\frac{k}{k+1} v^{2}\left(\frac{r_{0}}{r}\right)^{k+1}\right\} d x .
$$

Inserting equality (2.26) together with the last one into (2.24), we complete the proof.

Now we are in a position to prove the main result. Let $V^{0}:=\int_{\Omega} \eta^{0} d x$ be the initial volume.

Proposition 2.3. The following two-sided bounds for the gas volume hold

$$
\alpha_{1 \varepsilon} \mathscr{E}^{0} t+\beta_{1 \varepsilon} \leq V(t) \leq \alpha_{2 \varepsilon} \mathscr{E}^{0} t+\beta_{2 \varepsilon} \quad \text { for any } t \geq 0
$$

with any $0<\varepsilon<\nu_{1}$ and

$$
\begin{gathered}
\alpha_{1 \varepsilon}:=\frac{\min \left\{2 /(k+1), R / c_{V}\right\}}{\nu_{1}+\varepsilon}, \quad \alpha_{2 \varepsilon}:=\frac{\max \left\{2, R / c_{V}\right\}}{\nu_{1}-\varepsilon}, \\
\beta_{i \varepsilon}=\beta_{i \varepsilon}\left(V^{0}, \mathscr{E}^{0}, \nu, \mu, M, V_{0}\right), \quad i=1,2 .
\end{gathered}
$$


Proof. By virtue of the energy conservation law we have

$$
\begin{gathered}
\min \left\{\frac{2}{k+1}, \frac{R}{c_{V}}\right\} \mathscr{E}^{0} \leq \int_{\Omega}\left\{\frac{1}{k+1}\left[1+k\left(\frac{r_{0}}{r}\right)^{k+1}\right] v^{2}+R \theta\right\} d x \leq \max \left\{2, \frac{R}{c_{V}}\right\} \mathscr{E}^{0}, \\
\|v\|_{\Omega} \leq \sqrt{2 \mathscr{E}^{0}} .
\end{gathered}
$$

The latter bound and equality (2.6) together with the Young inequality imply

$$
\begin{aligned}
\left|\int_{\Omega} \frac{v}{r^{k}} I \eta d x\right| & \leq\|v\|_{L^{1}(\Omega)}\left\|\left(\frac{I \eta}{r^{k+1}}\right)^{k /(k+1)}\right\|_{C(\bar{\Omega})} V^{1 /(k+1)} \\
& \leq \sqrt{2 M^{\mathscr{C} 0}} \frac{1}{(k+1)^{k /(k+1)}} V^{1 /(k+1)} \\
& \leq \frac{1}{k+1}\left(\varepsilon_{0} V+c^{0} \varepsilon_{0}^{-1 / k}\right),
\end{aligned}
$$

with $c^{0}:=c_{0 k}\left(M_{\mathscr{C}^{0}}\right)^{(k+1) /(2 k)}$ and $c_{0 k}>0$ depending on $k$ only, for any $\varepsilon_{0}>0$. Therefore

$$
\left|W-v_{1} V\right| \leq \frac{1}{k+1}\left[\left(2 k|\mu| \varepsilon_{1}+\varepsilon_{0}\right) V+2 k|\mu| V_{0}\left(\left|\log V_{0}\right|+c_{\varepsilon_{1}}\right)+c^{0} \varepsilon_{0}^{-1 / k}\right],
$$

with $c_{\varepsilon_{1}}:=\log \left(\varepsilon_{1}^{-1}\right)+\varepsilon_{1}-1$, for any $\varepsilon_{1}>0$. This inequality remains valid for $W$ and $V$ replaced by $W(0)$ and $V^{0}$.

By integrating the key equality (2.21) and applying inequalities (2.34) and (2.37) with suitable $\varepsilon_{0}$ and $\varepsilon_{1}$ together with condition (1.8), we obtain the two-sided bounds (2.32).

Notice that the assumption $r_{0}>0$ has been not so crucial, the quantities $\beta_{i \varepsilon}$ in (2.32) are bounded as $r_{0} \rightarrow 0$ and thus the case without core, that is, $r_{0}=0$, could be also covered (at least for classical solutions) but we would not like to come into these details here.

\section{Acknowledgment}

The author is partially supported by the Russian Foundation for Basic Research, projects no. 04-01-00539 and 04-01-00619.

\section{References}

[1] S. N. Antontsev, A. V. Kazhikhov, and V. N. Monakhov, Boundary Value Problems in Mechanics of Nonhomogeneous Fluids, Studies in Mathematics and Its Applications, vol. 22, North-Holland, Amsterdam, 1990.

[2] H. Fujita-Yashima and N. Ablaoui-Lahmar, Sur l'expansion d'un gaz visqueux et calorifere avec la surface libre en une dimension et à symétrie sphérique [One-dimensional or spherically symmetric expansion of a heat-conducting viscous gas with free surface], Atti del Seminario Matematico e Fisico dell'Università di Modena 49 (2001), no. 1, 1-17.

[3] H. Fujita-Yashima and R. Benabidallah, Équation à symétrie sphérique d'un gaz visqueux et calorifere avec la surface libre [Spherically symmetric equation for a heat-conducting viscous gas 
8 The rate of the volume growth

with free surface], Annali di Matematica Pura ed Applicata. Serie Quarta 168 (1995), no. 1, 75117.

[4] O. A. Ladyženskaja, V. A. Solonnikov, and N. N. Ural'ceva, Linear and Quasi-Linear Equations of Parabolic Type, American Mathematical Society, Rhode Island, 1968.

[5] T. Nagasawa, On the asymptotic behavior of the one-dimensional motion of the polytropic ideal gas with stress-free condition, Quarterly of Applied Mathematics 46 (1988), no. 4, 665-679.

[6] A. Zlotnik, Asymptotic expansion of a one-dimensional viscous heat-conducting gas flow into halfspace, Dipartimento di Matematica Università di Torino, Quaderno 5 (2002), 1-27.

[7] _ Power-rate asymptotic expansion for viscous heat-conducting gas flows, to appear in Mathematical Models \& Methods in Applied Sciences (2006).

[8] A. Zlotnik and A. A. Amosov, Weak solutions to viscous heat-conducting gas 1D-equations with discontinuous data: global existence, uniqueness, and regularity, The Navier-Stokes Equations: Theory and Numerical Methods (Varenna, 2000) (R. Salvi, ed.), Lecture Notes in Pure and Appl. Math., vol. 223, Marcel Dekker, New York, 2002, pp. 141-158.

Alexander Zlotnik: Department of Mathematical Modelling, Moscow Power Engineering Institute, Krasnokazarmennaya 14, Moscow 111250, Russia

E-mail address: zlotnik@apmsun.mpei.ac.ru 


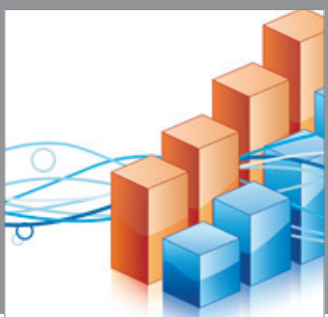

Advances in

Operations Research

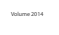

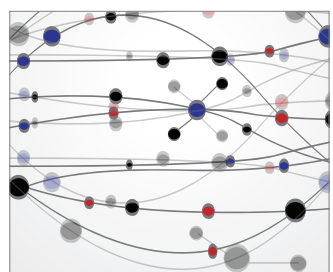

\section{The Scientific} World Journal
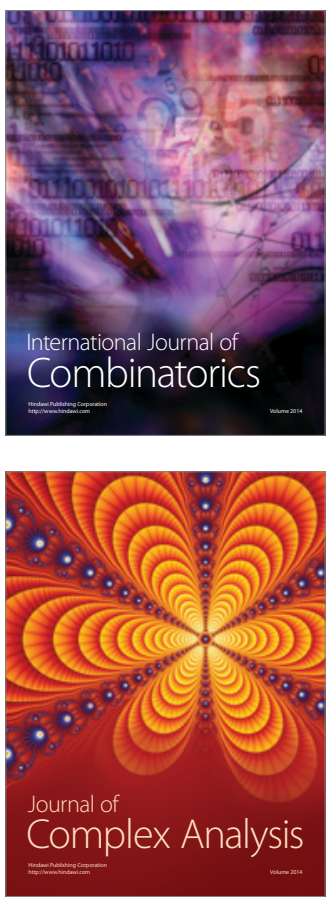

International Journal of

Mathematics and

Mathematical

Sciences
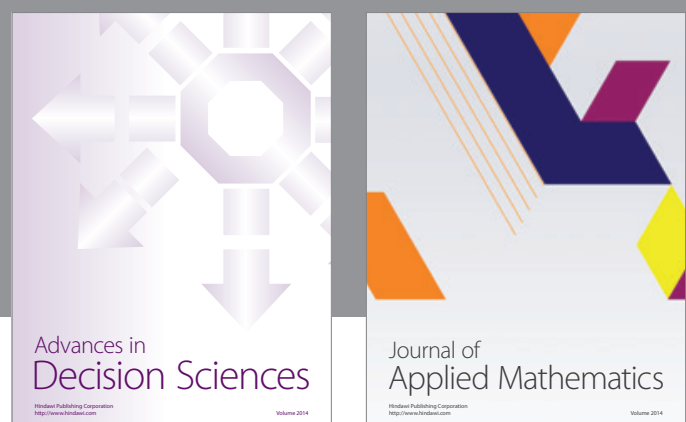

Journal of

Applied Mathematics
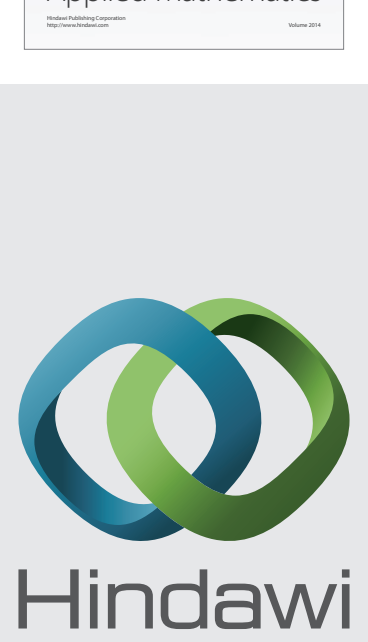

Submit your manuscripts at http://www.hindawi.com
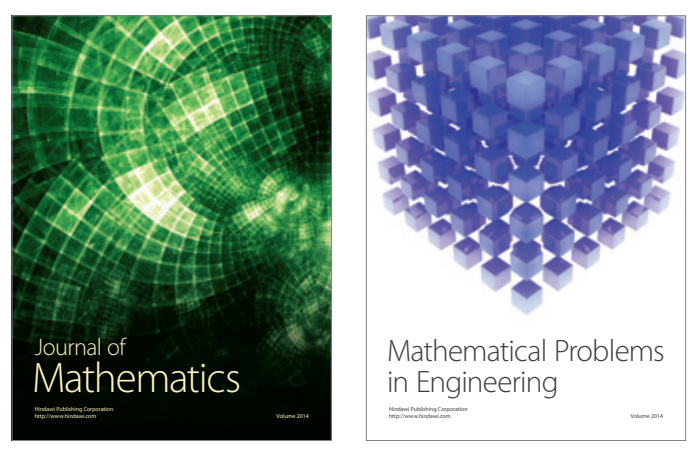

Mathematical Problems in Engineering
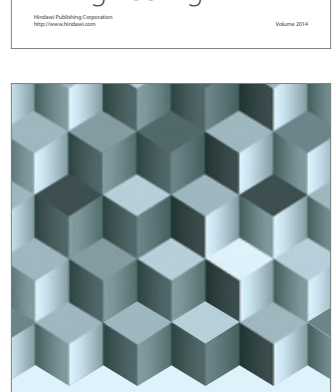

Journal of

Function Spaces
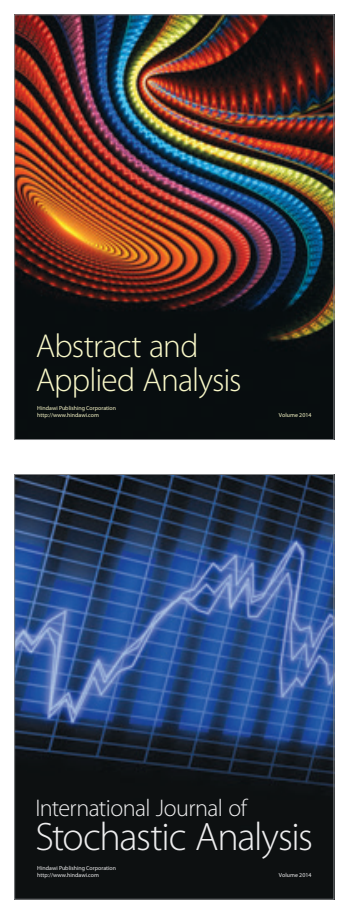

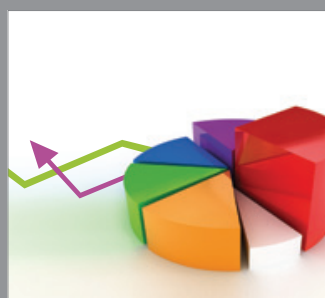

ournal of

Probability and Statistics

Promensencen
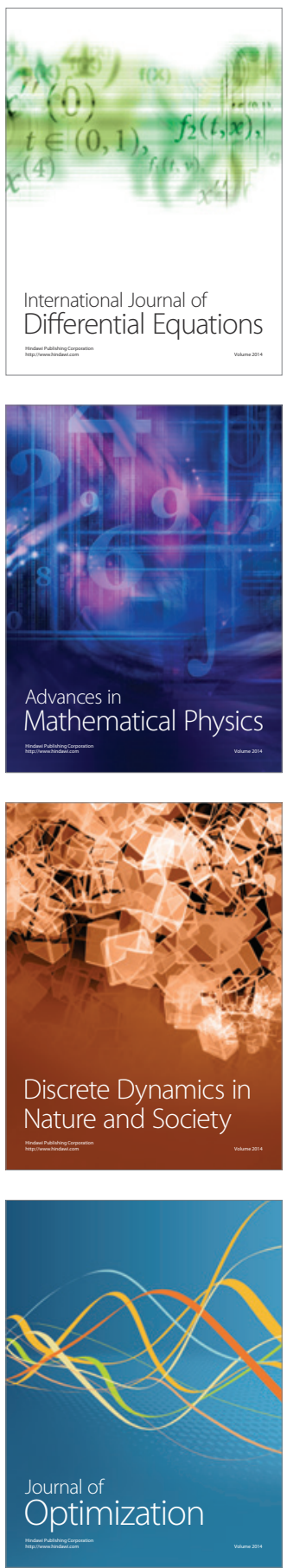